\title{
Effect of FLiBe thermal neutron scattering on reactivity of molten salt re- actor
}

\author{
Yafen $\mathrm{Liu}^{1}$, Wenjiang $\mathrm{Li}^{1, *}$, Rui Yan ${ }^{1, * *}$, Yang Zou ${ }^{1}$, Shihe $\mathrm{Yu}^{1}$, Bo Zhou ${ }^{1,2}$, and Xiangzhou Cai ${ }^{1}$ \\ ${ }^{1}$ Shanghai Institute of Applied Physics, CAS, Shanghai, 201800, China \\ ${ }^{2}$ University of Chinese Academy of Sciences, Beijing, 100049, China
}

\begin{abstract}
Thermal neutron scattering data has an important influence on the calculation and design of reactor with a thermal spectrum. However, as the only liquid fuel in the Gen-IV reactor candidates, the research on the thermal neutron scattering effect of coolant and somewhat moderator FLiBe has not been carried out sufficiently either experimentally or theoretically. The effect of FLiBe thermal neutron scattering on reactivity of TMSRLF (thorium molten salt reactor - liquid fuel), TMSR-SF (thorium molten salt reactor - solid fuel) and MSRE (molten salt reactor experiment) were investigated and compared. Results show that the effect of FLiBe thermal neutron scattering on reactivity depends to some extent on the fuel-graphite volume ratio of core. Calculations indicate that FLiBe thermal neutron scattering of MSRE (with the hardest spectrum) has the minimum effect of $41 \mathrm{pcm}$ on reactivity, and FLiBe thermal neutron scattering of TMSR-SF (with the softest spectrum) has the maximum effect of $-94 \mathrm{pcm}$ on reactivity, and FLiBe thermal neutron scattering of TMSR-LF has an effect of $-61 \mathrm{pcm}$ on reactivity at $900 \mathrm{~K}$.
\end{abstract}

\section{Introduction}

In a thermal neutron reactor, when neutron energy exceeds $4 \mathrm{eV}$, the binding energy within the molecule is not important in the process of neutrons colliding with the target nucleus [1]. It can be assumed that the target nucleus is stationary and unbound. However, it cannot be considered that the target nucleus is stationary when neutron energy is less than $4 \mathrm{eV}$ and is comparable with the heat energy of the nucleus. And when neutron collides elastically with the moving target nucleus, the energy of the outgoing neutron may be great than the energy of the incident neutron, which is the upward scattering phenomenon of neutron in the thermal region. In addition, there are interactions between scattered nucleus and neighboring nuclei in molecule or solid, so that nucleus is in a bound state and cannot recoil freely when colliding with neutrons. Therefore, the scattering cross section of the thermal energy region is not only changed with energy, but also related to the temperature and physical and chemical properties of the scattering medium [2, 3].

MSR (Molten Salt Reactor), using high temperature molten salt as fuel carrier and coolant, has three main advantages: excellent neutron economy, online reprocessing and inherent safety [4]. These make MSR an attractive research direction in the nuclear power industry. The fuel in MSR is dissolved in fluoride salt FLiBe $(67 \% \mathrm{LiF}$ $33 \% \mathrm{BeF}_{2}$ ), and it was considered that the thermal neutron scattering of $\mathrm{LiF}$ and $\mathrm{BeF}_{2}$ should be taken into consideration though they were not treated as moderators tradi-

\footnotetext{
*e-mail: liwenjiang@sinap.ac.cn

**e-mail: yanrui@sinap.ac.cn
}

tionally [5]. When neutrons are moderated to thermal, the binding of the scattering nucleus in a liquid moderator material affects the scattering cross section and the energy and angular distribution of secondary neutrons. The ability to accurately predict this process is significant for neutronics and safety in MSR.

This work aims at determining the effect of FLiBe thermal neutron scattering on reactivity of molten salt reactor. And three candidates were taken in to account: TMSRLF (thorium molten salt reactor - liquid fuel), TMSRSF (thorium molten salt reactor - solid fuel) and MSRE (molten salt reactor experiment). At first, the phonon spectra and the diffusion coefficients of FLiBe molten salt were obtained based on the first principle. Then, ENDF format thermal neutron scattering data of liquid FLiBe were generated by using LEAPR, and ACE format data were processed by NJOY99 subsequently [6]. At last, effects of FLiBe thermal neutron scattering on reactivity were investigated and compared in TMSR-LF, TMSR-SF and MSRE.

\section{Reactor Models}

TMSR-LF and TMSR-SF are the reactors chosen to be developed in the 'Thorium molten salt reactor (TMSR) nuclear energy system' project of the Strategic Priority Program launched by the Chinese Academy of Sciences in January 2011 [7, 8]. And the main features of these reactors are: using fluoride molten salt as fuel carrier and/or coolant, and moderated by graphite. $\mathrm{Nu}-$ clear fuel for TMSR-LF is ${ }^{235} U$ carried in a lithium-7flouride, beryllium-fluoride, and zirconium-fluoride and 
with or without thorium-fluoride salt. The mixture is fluid above $723 \mathrm{~K}$ (about $773 \mathrm{~K}$ for thorium-containing fuel) and has excellent flow, heat transfer properties and low vapor pressure. The salt is pumped into a closed loop through graphite moderator and is heated to about $913 \mathrm{~K}$ by fission of the fuel. The core housed in the reactor vessel is made up from top to bottom of upper graphite packing layer, upper plenum and pressure plate, active region, bottom plate, lower plenum and lower graphite packing layer. At room temperature, the core is approximately $237.0 \mathrm{~cm}$ in diameter and $320.0 \mathrm{~cm}$ in height. The active region of the core is located between the upper and bottom plates, composed of graphite component with channels and the fuel flowing through them. There are 244 channels in the graphite component including fuel channels and functional channels. The diameter of the fuel channels is 4.0 $\mathrm{cm}$ and the center to center spacing is $10.0 \mathrm{~cm}$.

TMSR-SF is a fluoride salt-cooled high-temperature reactor (FHR) using coated particle fuel and FLiBe as its primary coolant. The pebbles in the TMSR-SF are $6 \mathrm{~cm}$ in diameter and Tri-structural isotropic (TRISO) particles are made up of $17.0 \%$ enriched $U_{2}$ kernels coated with a low-density buffer layer, an inner pyro lytic carbon layer, an intermediate silicon carbide layer and an outer pyro lytic carbon layer. The description and parameter are detailed in Reference [9].

MSRE is recognized as the representative of molten salt reactor, which was fueled with ${ }^{235} U$ or ${ }^{233} U$ using fluorine salt as carrier salt and moderated with graphite. It was used to be compared with TMSR-LF and TMSR-SF on the effect of the FLiBe thermal neutron scattering on reactivity. Models for these three reactors are shown in Figure $1[10]$. FLiBe molten salt are used in these three
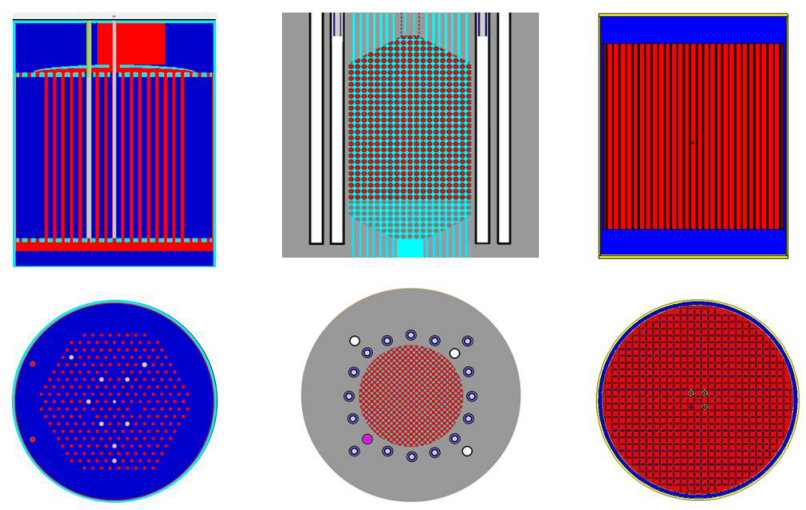

Figure 1. Vertical and horizontal cut in TMSR-LF (left), TMSRSF (middle) and MSRE (right) by MCNP5.

reactors as fuel carrier or coolant and the slow-down properties of it is list in Table 1, where lithium is ${ }^{7} L i$ and beryllium is ${ }^{9} \mathrm{Be}$. All the parameters in this table are calculated by using the data of $0.0253 \mathrm{eV}$ in ENDF/B-VII.0 [11]. It is concluded that the moderating effect of FLiBe cannot be neglected by comparing with this effect of graphite.
Table 1. The slow-down properties of FLiBe and graphite.

\begin{tabular}{llll}
\hline Material & Lethargy $\xi$ & $\xi \Sigma_{s}, c m^{-1}$ & $\xi \Sigma_{s} / \Sigma_{a}$ \\
\hline Li in $L i F$ & 0.26 & 0.06 & 5.7 \\
$\mathrm{~F}$ in $L i F$ & 0.102 & 0.017 & 40 \\
Be in $B e F_{2}$ & 0.207 & 0.053 & 144 \\
Graphite & 0.158 & 0.085 & 220 \\
\hline
\end{tabular}

\section{Methodology}

The thermal neutron scattering cross section is usually divided into three parts. 1) Inelastic scattering: including coherent and incoherent inelastic scattering, which is important for all materials, is represented by the scattering law $S(\alpha, \beta)$. 2) Incoherent elastic scattering: it is important for solids containing hydrogen, such as zirconium hydride, polyethylene, solid light water, etc. 3) Coherent elastic scattering: important for crystals, such as graphite, beryllium and so on. The thermal scattering cross section can be written as:

$$
\sigma\left(E \rightarrow E^{\prime}, \mu\right)=\frac{\sigma_{b}}{2 \kappa T} \sqrt{\frac{E^{\prime}}{E}} S(\alpha, \beta)
$$

where $E$ and $E^{\prime}$ are the incident and outgoing neutron energy, $\mu$ is the cosine of the scattering angle, $\sigma_{b}$ is the characteristic bound scattering cross section of material, and $\kappa T$ is thermal energy in $\mathrm{eV}$ [1]. $S(\alpha, \beta)$ is computed using phonon spectrum, which is performed by LEAPR module of NJOY. And the phonon spectrum of FLiBe needed in this work were obtained based on the first principle [12]. The data processing flow is shown in Figure 2. And the processed thermal scattering cross sections are then provided to MCNP5 code for the final criticality and reactivity calculations [10].

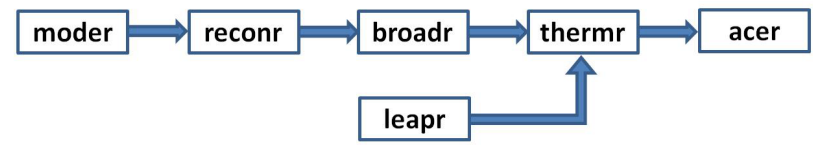

Figure 2. Flow diagram of thermal neutron scattering data processing.

\section{Phonon Spectrum and Scattering Cross Section}

Based on the first principle, the high-precision quantum chemical calculation method was developed, and the deduced all-atom polarization field was applied to FLiBe molten salt. In order to simulate the molten salt system as truly as possible, isothermal and isobaric simulation of NVT ensemble was adopted in this work. In the process of molecular dynamics simulation, the temperature was controlled by the Nose Hover weak coupling method, and the coupling constant was set as 0.05 ps [12]. When the initial configuration was established, in order to avoid the overlap of atoms, a larger box was initially selected, and then the system was gradually compressed to the liquid density by NPT ensemble. Then the system was completely random in liquid state by simulated annealing. All 

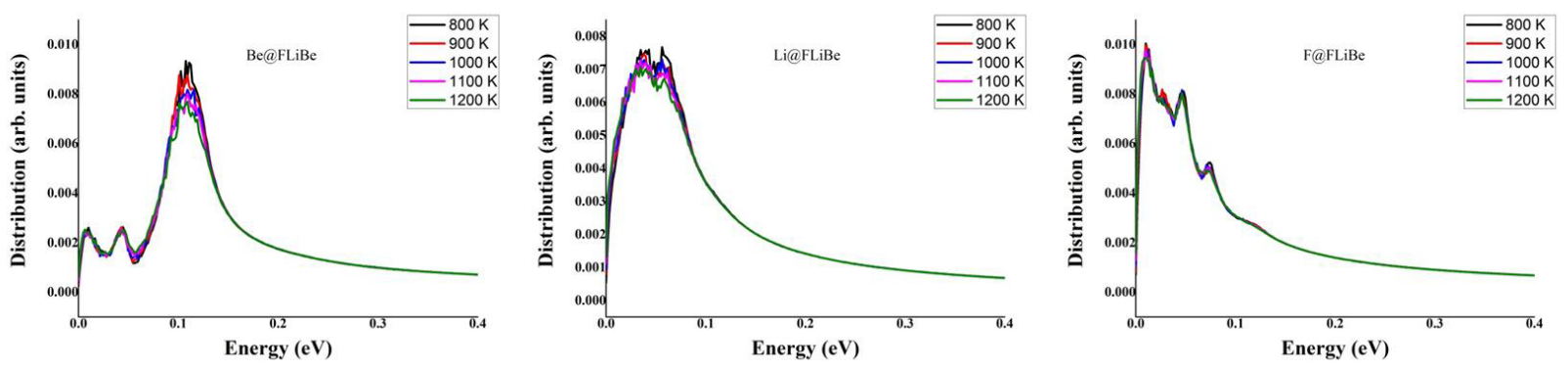

Figure 3. Phonon spectra of $B e^{2+}$ (left), $\mathrm{Li}^{+}$(middle) and $\mathrm{F}^{-}$(right) in FLiBe molten salt.

the molecular dynamics simulation processes were implemented by $\mathrm{CP} 2 \mathrm{~K}$ simulation program. Figure 3 shows the phonon spectra of beryllium, lithium and fluoride in FLiBe molten salt at different temperatures. The peaks of the photon spectra are at the energy of $0.1 \mathrm{eV}$ for beryllium, and $0.04 \mathrm{eV}$ and $0.06 \mathrm{eV}$ for lithium, and $0.04 \mathrm{eV}$ for fluoride. What's more, it is concluded that FLiBe molten salt has only inelastic scattering, and the inelastic scattering cross section decreases with the increase of temperature.

Figure 4 shows the final inelastic scattering cross sections of beryllium, lithium and fluoride in FLiBe molten salt at different temperatures. The influence of different translational weight $(0.01$ and 0.05$)$ on thermal neutron scattering libraries for FLiBe was tested. The inelastic scattering cross section decreases with the increase of temperature, and decreases with the increase of translation weight. In fact, the increase of temperature and the increase of transfer weight are both processes of the increase of atomic migration capacity in the liquid, that is to say, the increase of migration capacity will reduce the inelastic scattering cross section.

\section{Results and Conclusion}

Table 2 shows the effect of FLiBe thermal neutron scattering on $k_{e f f}$ of TMSR-LF, TMSR-SF and MSRE and Figure 5 shows the reactivity change due to the consideration of this reaction. Different translational weights have little influence on $k_{\text {eff }}$ (less than 0.00050), so the weight of 0.05 was chosen in this work. It is concluded that FLiBe thermal neutron scattering of MSRE has the minimum effect of $41 \mathrm{pcm}$ on reactivity, and TMSR-SF has the maximum effect of $-94 \mathrm{pcm}$ on reactivity, and TMSR-LF has an effect of $-61 \mathrm{pcm}$ on reactivity at $900 \mathrm{~K}$. And the effect is less than that of graphite, which is about $-200 \mathrm{pcm}$ for TMSR-LF and MSRE, and about 100 pcm for TMSR-SF.

Figure 6 shows the neutron spectra in the molten salt of these three reactors. As can be seen from this figure that the spectrum of MSRE is the hardest and the spectrum of TMSR-SF is the softest among these three reactors. It is indicate that the influence of thermal scattering on reactivity is mainly depending on the energy spectrum and the softer the energy spectrum, the greater the influence of thermal scattering.

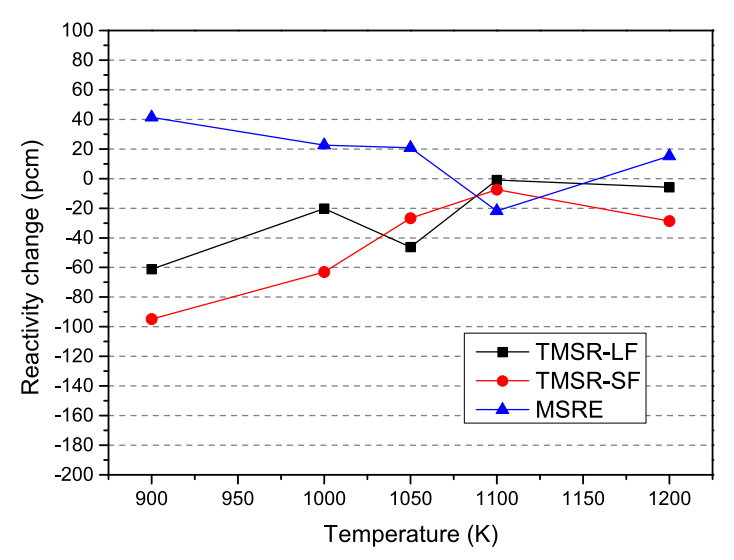

Figure 5. Neutron spectrum comparison between TMSR-LF, TMSR-SF and MSRE.

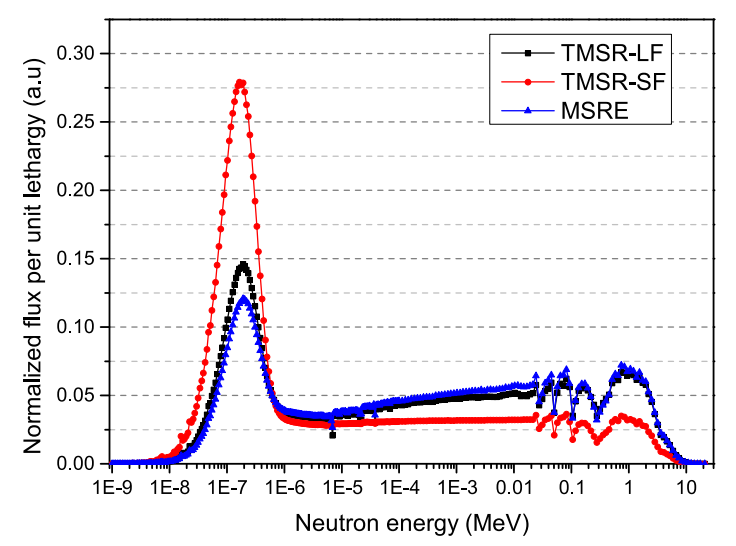

Figure 6. Reactivity change due to the consideration of FLiBe thermal neutron scattering.

\section{Acknowledgements}

This study was supported by the Chinese TMSR Strategic Pioneer Science and Technology Project (No.XDA02010000), the Frontier Science Key Program of Chinese Academy of Sciences, China (No.QYZDY-SSW- 

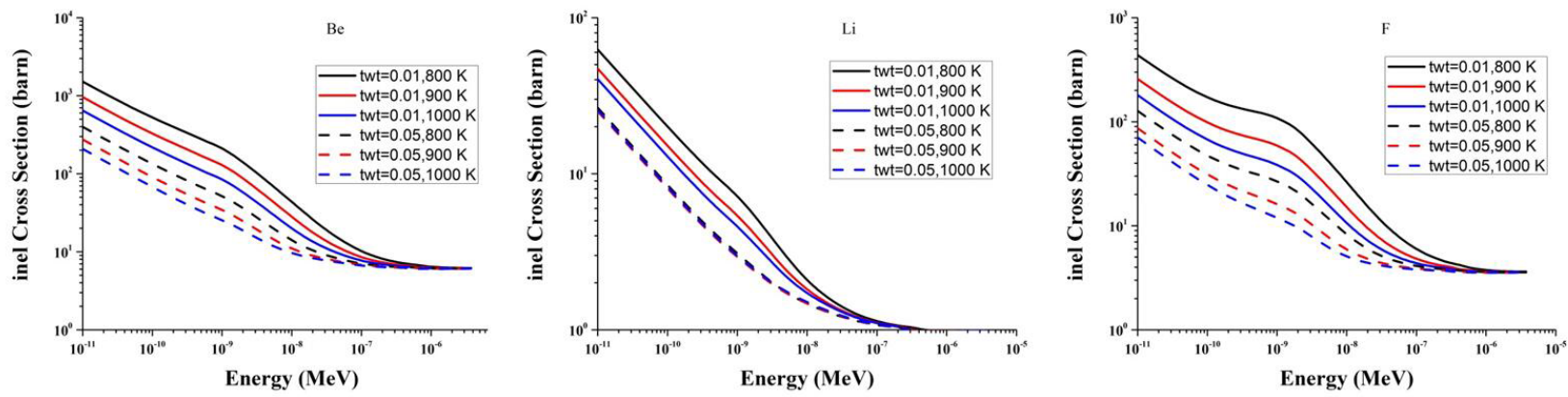

Figure 4. Inelastic scattering cross sections of $\mathrm{Be}^{2+}$ (left), $\mathrm{Li}^{+}$(middle) and $\mathrm{F}^{-}$(right) in FLiBe molten salt.

Table 2. The effect of FLiBe thermal neutron scattering on $k_{\text {eff }}$.

\begin{tabular}{lcccccc}
\hline & \multicolumn{2}{c}{ TMSR-LF } & \multicolumn{2}{c}{ TMSR-SF } & \multicolumn{2}{c}{ MSRE } \\
\hline & $\begin{array}{c}k_{\text {eff }} \\
\text { without FLiBe } \\
\text { therm. scat. }\end{array}$ & $\begin{array}{c}k_{\text {eff }} \text { with FLiBe } \\
\text { therm. scat. }\end{array}$ & $\begin{array}{c}k_{\text {eff }} \\
\text { without FLiBe } \\
\text { therm. scat. }\end{array}$ & $\begin{array}{c}k_{\text {eff }} \\
\text { with FLiBe } \\
\text { therm. scat. }\end{array}$ & $\begin{array}{c}k_{\text {eff }} \\
\text { without FLiBe } \\
\text { therm. scat. }\end{array}$ & $\begin{array}{c}k_{\text {eff }} \text { with FLiBe } \\
\text { therm. scat. }\end{array}$ \\
900 & 1.02309 & 1.02245 & 1.04272 & 1.04169 & 1.03200 & 1.03244 \\
1000 & 1.01965 & 1.01944 & 1.04185 & 1.04120 & 1.03064 & 1.03088 \\
1050 & 1.01844 & 1.01796 & 1.04105 & 1.04076 & 1.02954 & 1.02976 \\
1100 & 1.01647 & 1.01646 & 1.04078 & 1.04070 & 1.02785 & 1.02762 \\
1200 & 1.01376 & 1.01370 & 1.03995 & 1.03964 & 1.02570 & 1.02586 \\
\hline
\end{tabular}

JSC016) and Shanghai Sailing Program with grant/award Number: Y931021031.

\section{References}

[1] Mei L, Cai X, Jiang D, et al. The investigation of thermal neutron scattering data for molten salt Flibe, Journal of Nuclear Science Technology 50, 682-688 (2013)

[2] Chen C, Chen Y, Hu Z, et al. Generating and Validation of Thermal Neutron Scattering Library for MCNP, Atomic Energy Science and Technology (in Chinese) 44, 1335-1340 (2010)

[3] Wang L, Jiang X, Zhao Z, et al. Investigation of thermal neutron scattering cross sections for $\mathrm{LiH}$, Nuclear Engineering and Design 262, 365-370 (2013)

[4] Yafen L, Rui Y, Yang Z, et al. Criticality Properties and Control Rod Worth of the Critical Experiment Device for MSR Research, Nuclear Technology 2018, 1-10 (2018)

[5] W.K. Ergen, A.D. Callihan, C.B. Mills, Dunlap Scott. The Aircraft Reactor Experiment-Physics, Nuclear Science and Engineering 2, 826-840 (1957)

[6] MacFarlane, R.E., Muir, D.W.,1999. NJOY99: Code system for Producing Point Wise and Multigroup Neu- tron and Photon Cross sections from ENDF/B. RSICC code package PSR-480. Los Alamos National Laboratory, Los Alamos, New Mexico,USA

[7] Yafen L, Rui Y, Yang Z, et al. Neutron flux distribution and conversion ratio of Critical Experiment Device for molten salt reactor research, Annals of Nuclear Energy 133, 707-717 (2019)

[8] Xu H, Dai Z, Cai X. Some Physical Issues of the Thorium Molten Salt Reactor Nuclear Energy System, Nuclear Physics News 24, 24-30 (2014)

[9] Rui-Min Ji, Li M H , Zou Y, et al. Impact of photoneutrons on reactivity measurements for TMSR-SF1, Nuclear Science and Techniques 28, 101-107 (2017)

[10] X-5 Monte Carlo Team, 2003. MCNP C a general N-particle transport code, Version 5, LA-UR-03C1987, Los Alamos National Laboratory

[11] Chadwick M B, Oblozinsky P, Herman M, et al. ENDF/B-VII.0: Next Generation Evaluated Nuclear Data Library for Nuclear Science and Technology, Nuclear Data Sheets 107, 2931-3060 (2017)

[12] Dai J , Han H , Li Q , et al. First-principle investigation of the structure and vibrational spectra of the local structures in LiFCBeF2 Molten Salts, Journal of Molecular Liquids 213, 17-22 (2016) 